\title{
A rendezvous approach for correcting accumulative errors of multiple robots
}

Xuefeng Dai ${ }^{1 *} \mathbb{D}$, Zheping Yan², Jianqi Zhao ${ }^{1}$ and Dahui $\mathrm{Li}^{1}$

\begin{abstract}
Wireless communication with no range and bandwidth limitations is desired for coordination and information sharing among multiple robots. However, the perfect communication is not available for a few of reasons. This paper proposed a simple yet effective scheme for correcting odometer errors existed in each robot of a multi-robot system. A contribution is that additional communication bandwidth is needed only if a rendezvous for two robots happened. Implementation of the error correction scheme is addressed in detail. Moreover, rendezvous is formulated by a set of predicate logic reasoning implications for each robot at upper level of soft architecture. The proposed approach was validated by computer simulations.
\end{abstract}

Keywords: Error correction, Rendezvous, Predicate logic reasoning, Multi-robot systems, Exploration

\section{Introduction}

Multi-robot systems not only outperform single robot systems in redundancy and robustness, but also can finish tasks that the single robot systems cannot do. There are many applications for multi-robot systems in dangerous, poisonous, and radioactive operation environments. The goal for the multi-robot systems to achieve during operational discourse varies according to applied scenarios. For example, the multiple robots try to scan unknown environments in a minimal time for a search and rescue application. Therefore, the corresponding coordinated strategy emphasized on minimizing repeated coverage and task balancing across robots. Lots of results are available to deal with the subject [1-4]. However, uncertainties which are intrinsic in sensing and acting for all robots have not been addressed in the above results. A typical uncertainty is odometer error accumulated during exploration [5]. Simultaneous localization and mapping algorithms can overcome the uncertainty by fusing estimates provided by both sensory matching and dead reckoning techniques [6, 7]. Unfortunately, if observations are not available, i.e., all environmental features are out of sensing range of the robot, then the algorithms fail to overcome the uncertainty. Markov decision process (MDP) modeling-based

\footnotetext{
*Correspondence: daixuefeng203@163.com

${ }^{1}$ School of Computer and Control Engineering, Qiqihar University, Qiqihar

161006, Heilongjiang, China

Full list of author information is available at the end of the article
}

approaches for multi-robot systems coordination took the uncertainty into account [8-11]. Yet, solving MDP are computational expensive. In general, there are no analytic solutions to MDP. On the other hand, the coordination depends on wireless communication with finite range and finite bandwidth. Therefore, a practical odometer error correction scheme should not overload the bandwidth of the network. Such a novel uncertainty correction scheme which can be embedded in predicate logic reasoning-based approach (PLR-A) [12] for multi-robot systems exploring unknown environments is proposed in this paper.

It is hard to consider all variables about teammate robots and operational environment when selecting navigation goal for a specific teammate robot. So, PLR-A was proposed by the authors of this paper. The work realized cooperative exploration by predicate logic reasoning in a partitioned state space instead of a joint state space for all robots and the environment. However, the uncertainties were not addressed there. This paper discusses the uncertainty correction by position information fusion through rendezvous of any two teammate robots. A contribution of this paper is odometer error that is corrected by taking advantage of rendezvous. Another contribution is that additional communication bandwidth is needed only if a rendezvous happened. Moreover, formal expression of rendezvous at upper level is expressed by predicate logic reasoning in the adopted hierarchical architecture. 
This paper is organized as follows. Section 2 reviews related work. Section 3 presents preliminary for exploration through a special kind of zigzag trajectories. Section 4 discusses error correction scheme through rendezvous and filtering of position information. Section 5 gives simulation settings for validating the proposed approach. Section 6 presents computer simulation and statistical results and discussions about the results. Finally, Section 7 concludes the paper.

\section{Related work}

Coordinated algorithms take roles of task assignments to robots, robots dispersion over operational environments, and cooperation across robots. They make all robots of a multi-robot system working together as a whole. Coordination has been an active subject in the field of intelligent robotics for two decades. Study achievements about distributed multi-agent coordination since 2006 were reviewed from systems and control point of view [13]. Methods for coverage path planning during the past decade of 2013 were reviewed [14]. In addition, the work also reviewed coverage under uncertainty and multi-robot coverage methods.

Recently, a few of new trends appeared, for example, energy-constrained coordination [2], joint exploration and tracking [3], and coordination under recurrent communication constraints [4]. An energy-constrained spatiotemporal rendezvous problem of multi-robot systems was discussed [2]. It was formulated as an optimization problem with a global cost function and local constraints, where all robots were minimizing a same objective function, but each robot had its own constraint. Multi-robot multi-object exploration and tracking problem was solved simultaneously by expressing it as an optimal control problem that is to maximize objective function subject to dynamics and detection probability [3]. A hierarchical formulation was presented in the work, and the continuous optimal control problem for each robot was solved independently by a nonlinear optimization algorithm at lower level. Finally, exploration strategies that allow robots to coordinate with teammates to form an ad hoc network to satisfy recurrent connectivity constraints were designed [4].

A major part of results that addressed the uncertainties are MDP and its variants modeling-based approaches. Typical results included and emphasized on sequential decision-making $[8]$ and coordination $[9,10]$ under uncertainties. The problem of sequential decision-making under uncertainties for each robot was formulated as a MDP [8]. A decentralized model learning scheme based on the Incremental Feature Dependency Discovery (Dec-iFDD) was presented to learn uncertain state transition model in the underlying MDP. The scheme shared the most relevant features across teammates under limited communication. Moreover, a macro-action decentralized partially observable MDP (MacDec-POMDP)-based method for multi-robot coordination was introduced in the presence of uncertain sensing and other limitations [9]. A new planning algorithm which was called MacDec-POMDP heuristic search was presented. To overcome the issue of information space scales exponentially with the number of robots, the multi-robot POMDP was decentralized by POMDP policy auctions [10]. Moreover, a problem of assigning a set of stationary targets to a team of mobile robots under temporal, performance, perception, and resource constraints was addressed [11]. The corresponding constrained MDP was solved in two steps. Another related work is exploration plan based on stochastic dynamic programming [15] in which uncertainties in both sensing and control were taken into account. However, it is hard to solve MDP and its variants.

Furthermore, collaborative localization [16] and active information gathering [17] under uncertainty were discussed. A synthesizing method for multi-robot collaborative localization was described [16]. In the method, an odometry and a North Star positioning system were used to perform localization for each robot. Then, a distributed extended Kalman filter and relative observation combining approach were outlined. Moreover, a problem of reducing uncertainty about a physical process of interest by designing sensing trajectories for a team of robots was considered [17]. An anytime planning algorithm progressively reduced suboptimality of the information gathering plans.

On the other hand, rendezvous played important roles in multi-robot systems, for example, it was used to realize collaboration [1], recharging [18], exploration [19], and formation flight [20]. There is a case that require robots to collaboratively perform a physical action at a common position. For example, the case that doors maybe closed or paths maybe blocked in search and rescue mission after a disaster, so collaboration (rendezvous) of robots is needed [1]. Recharging issue which was represented as a rendezvous problem of a group of mobile robots in their working environment had been addressed [18]. It had been proven that orthogonal least absolute value regression-based planner resulted to a route that minimized cumulative sum of worker robot distances traveled by all robots during the recharging process. Since robot rendezvous is a key step in collaborative exploration, two algorithms that robots could use in attempting to rendezvous quickly were proposed [19]. The first is a deterministic one that created a list of all combination of landmarks and specified the order in which the landmarks should be visited. One robot picked a landmark and waited there for the other robot. The second is a probabilistic one that simply generated probabilities for landmarks being visited. Besides mobile robot rendezvous, unmanned aerial vehicle (UAV) rendezvous is a necessary precondition for formation flight [20]. Time-varying vector fields were designed to adjust trajectories according to estimated flight time; 
therefore, the UAVs can reach rendezvous position at the same time.

Different with above results, the rendezvous is used to fuse location coordinate information in the proposed approach. In addition, predicate logic reasoning is used to formulate the rendezvous at upper level. To the best of our knowledge, this is the first try to discuss error correction under communication constraints based on rendezvous and reasoning. Predicate logic reasoning has been used in automatic composition of semantic Web services. For example, fuzzy predicate Petri net was proposed to model Horn clauses [21]. Moreover, reasoning has been used in ontology technology which is one of the most promise methodologies to express and propagate knowledge [22].

\section{Preliminary}

It is assumed in this paper that a multi-robot system $S$ $=\left\{r_{1}, \ldots, r_{n}\right\}$ is considered, where $n$ is the size of the system. All robots are equipped with vision or sonar sensors for the purposes of scanning and recognizing each other when two robots are sufficiently close together. In addition, an instantaneous, infinite bandwidth communication between robots at all times are available. If each mobile robot of the system was taken as a mobile node, then all nodes constitute a full-connected wireless network.

For convenience of comprehension, some results related to predicate logic reasoning-based coordination of multi-robot systems [12] are reviewed in brief and some results are further addressed in the following.

First, it was assumed that all robots explore an operational environment through a kind of modified zigzag trajectories. A few of concepts about the zigzag trajectories will be reviewed. The zigzag trajectory consists of a series of shorter line segment sub-trajectories and longer line segment sub-trajectories. The direction that corresponds to the shorter line segment is named as primary exploring direction, and the direction that corresponds to the longer line segment is named as auxiliary exploring direction. The distance between two adjacent trajectory line segments of auxiliary exploring directions of a robot $r_{i}(i=1, \ldots, n)$ is named as width of $r_{i}^{\prime}$ s zigzag trajectory.

The exploration coordination consisted of partitioning the multi-robot system, initialization of exploration discourse, and predicate logic reasoning for coordination. Before exploration begins, the multi-robot system was partitioned into at most three subsets. The partitioning first aimed at making the size of each subset as an even number as possible, second aimed at making the number of subsets as small as possible and the least size of the first subset is 2 , and finally aimed at making the sizes of the three subsets as equivalent as possible. Robots belonged to the same subset are named as cooperative robots. After the partitioning, exploration discourse of the multi-robot system would be initialized. Initialization strategies for a multi-robot system with $n=5$ are shown in Fig. 1 where the partitioning is $S=\left\{\left\{r_{1}, r_{2}\right\},\left\{r_{3}, r_{4}\right\},\left\{r_{5}\right\}\right\}$.

Figure 1a, $\mathrm{b}$ shows the initializing processes for cases that robots cannot sense boundary and can sense right boundary of operational environment at initial locations, respectively. All robots start their exploration discourses from a common initial location where it is marked as a circle in the figures. It is assumed that auxiliary exploring directions are vertical. Then, $r_{1}, r_{3}$, and $r_{5}$ move upwards, and $r_{2}$ and $r_{4}$ move downwards in the beginning of exploration. As soon as the robots sensed upper boundary or lower boundary, they change to their exploring directions to their primary exploring directions, as shown in the figures.

A feature of modified zigzag trajectories is that two robots belonged to the same subset will have at least one rendezvous for the case shown in Fig. 1. Having moved a distance of their zigzag trajectory widths on their primary exploring directions, two cooperative robots began to move to each other from opposite directions on auxiliary exploring directions during the exploration discourse. As shown in Fig. $1, r_{1}$ and $r_{2}$, and $r_{3}$ and $r_{4}$ will have a few of rendezvous, respectively. It is not sure that $r_{5}$ will not have rendezvous along its auxiliary or primary exploring directions since it has no cooperative robot. In addition, initialization

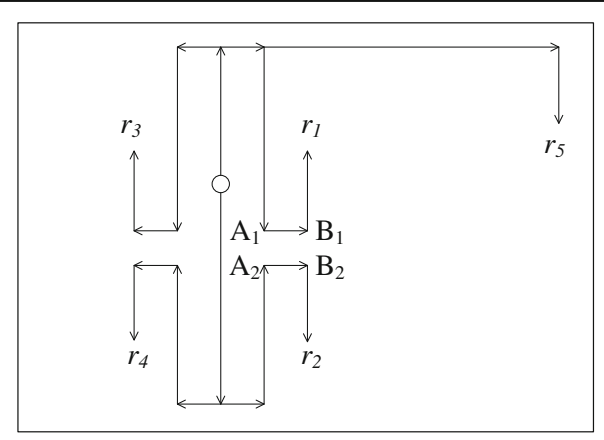

(a) Robots cannot sense boundary

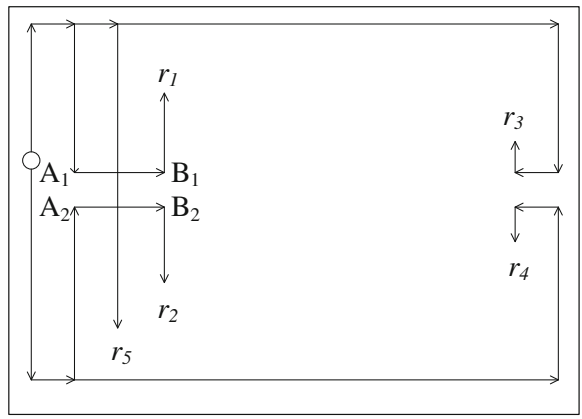

(b) Robots can sense right boundary

Fig. 1 Initialization for exploration discourse of a multi-robot system $(n=5)$; in the figure, $r_{j}$ represents robot $i(i=1, \ldots, 5), A_{j}$ and $B_{j}$ represent rendezvous start and end locations of $r_{j}(j=1,2)$, respectively, and all arrows represent moving directions of the corresponding robots 
strategies for a multi-robot system with $n=6$ are shown in Fig. 2 where the partitioning is $\boldsymbol{S}=\left\{\left\{r_{1}, r_{2}\right\},\left\{r_{3}, r_{4}\right\},\left\{r_{5}, r_{6}\right\}\right\}$.

In the following, $r_{1}$ and $r_{2}$ are taken as examples to discuss rendezvous. Supposed that $r_{1}$ arrived at $\mathrm{A}_{1}$ and $r_{2}$ arrived at $\mathrm{A}_{2}$ where $r_{1}$ has recognized $r_{2}$, or $r_{2}$ has recognized $r_{1}$, or both robots have recognized each other, then a rendezvous happened. The time period for two robots from meeting to separating is named as rendezvous period. The distance $\mathrm{A}_{i} \mathrm{~B}_{i}(i=1,2)$ traveled by the two robots during the rendezvous period is named as rendezvous distance. The distance is equal to $2 d_{i}$ and $4 d_{i}$, respectively in Fig. $1 \mathrm{a}, \mathrm{b}$.

It is remarked that meeting locations have no dependence on the environments-every location is a potential rendezvous location.

\section{Uncertainty correction scheme}

If two robots $r_{i}$ and $r_{j}(i, j=1, \ldots, n, i \neq j)$ belonged to the same subset $S_{k}$, and they have moved to each other along their auxiliary exploring directions and were sufficient close to recognize each other, then the formal expression of the rendezvous at upper level by predicate logic reasoning is

$$
\begin{aligned}
& \text { Belonged }\left(r_{i}, S_{k}\right) \wedge \text { Belonged }\left(r_{j}, S_{k}\right) \wedge \operatorname{Moved}\left(r_{i}, \text { dir }_{i}\right) \wedge \text { Moved } \\
& \left(r_{j}, \operatorname{dir}_{j}\right) \wedge B e_{-} \text {oppst }\left(\text { dir }_{i}, \text { dir }_{j}\right) \wedge B e_{-} \text {close }\left(r_{i}, r_{j}\right) \wedge \\
& \left(\operatorname{Rcgnz}\left(r_{i}, r_{j}\right) \vee \operatorname{Rcgnz}\left(r_{j}, r_{i}\right)\right) \Rightarrow \text { Fuse }\left(\operatorname{Coord}_{x}\left(r_{i}\right), \operatorname{Coord}_{x}\right. \\
& \left.\left(r_{j}\right)\right) \wedge \text { Fuse }\left(\operatorname{Coord}_{y}\left(r_{i}\right), \operatorname{Coord}_{y}\left(r_{j}\right)\right)
\end{aligned}
$$

where Belonged, Moved, Be_oppst, Be_close, Rcgnz, Coord $_{x}$, and Coord $y_{y}$ are first order predicates, and Fuse is a second order predicate, and $r_{i}, S_{k}$, and $d i r_{i}$ are variables, all of these predicates and corresponding variables are commentated in Table 1.

During the rendezvous period, coordinate $x$ of the two robots $r_{i}$ and $r_{j}$ are kept to be identical, Fuse $\left(\operatorname{Coord}_{x}\left(r_{i}\right)\right.$, $\left.\operatorname{Coord}_{x}\left(r_{j}\right)\right)$ is conducted iteratively by

$$
\begin{aligned}
& \Delta x_{i j}(k+1)=\frac{\Delta x_{i}(k+1)+\Delta x_{j}(k+1)}{2} \\
& x_{i j}(k+1)=x_{i j}(k)+\Delta x_{i j}(k+1)\left(k=0, \ldots, k_{r}-1\right)
\end{aligned}
$$

where $x_{i j}(k+1)$ is coordinate $x$ at time instant $k+1$ of the two robots; $\Delta x_{i}(k+1)$ and $\Delta x_{j}(k+1)$ are coordinate increments of $r_{i}$ and $r_{j}$, respectively, which are derived from dead reckonings; $\Delta x_{i j}(k+1)$ is a fused increment; and $k_{r}$ is the rendezvous period. When $k=0, x_{i j}(0)$ $=\left(x_{i}(0)+x_{j}(0)\right) / 2$, and $y_{i}(0)=\left(y_{i}(0)+y_{j}(0)\right) / 2+d_{i j} / 2$ and $y_{j}(0)=\left(y_{i}(0)+y_{j}(0)\right) / 2-d_{i j} / 2$. The fusion for coordinate $y_{i}$ and $y_{j}$ are realized by

$$
\begin{aligned}
& \Delta y_{i j}(k+1)=\frac{\Delta y_{i}(k+1)+\Delta y_{j}(k+1)}{2} \\
& y_{i}(k+1)=y_{i}(k)+\Delta y_{i j}(k+1) \\
& y_{j}(k+1)=y_{i}(k+1)-d_{i j}\left(k=0, \ldots, k_{r}-1\right)
\end{aligned}
$$

where $d_{i j}=\left|\mathrm{A}_{1} \mathrm{~A}_{2}\right|$ is the distance between the two robots when they met. During the rendezvous period, $d_{i j}$ will be kept as a constant. A rendezvous is over if and only if

$$
\begin{array}{r}
\text { Belonged }\left(r_{i}, S_{k}\right) \wedge \text { Belonged }\left(r_{j}, S_{k}\right) \wedge \text { Moved }\left(r_{i}, \text { dir }_{i}\right) \wedge \text { Moved } \\
\left(r_{j}, \text { dir }_{j}\right) \wedge \neg \text { Be_oppst }\left(\text { dir }_{i}, \text { dir }_{j}\right) \wedge \operatorname{Travelled}_{(}\left(r_{i}, d_{r}\right) \wedge \\
\text { Travelled }\left(r_{j}, d_{r}\right) \Rightarrow \text { Fuse }\left(\text { Coord }_{x}\left(r_{i}\right), \text { Coord }_{x}\left(r_{j}\right)\right) \wedge \neg \text { Fuse } \\
\left(\text { Coord }_{y}\left(r_{i}\right), \text { Coord }_{y}\left(r_{j}\right)\right)
\end{array}
$$

where $d_{r}$ is the rendezvous distance. If $r_{i}$ and $r_{j}$ keep current primary exploring directions, $d_{r}$ is equal to the width of zigzag trajectory; otherwise, $d_{r}$ is equal to a distance commonly traveled by the two robots until they could sense unexplored area.

For two robots belonged to different subsets $S_{k} \in S$ and $S_{m} \in S(k \neq m)$, they will fuse their coordinates information also by (2) to (6) when they met on their auxiliary exploring directions. A difference with cooperative robots is that one

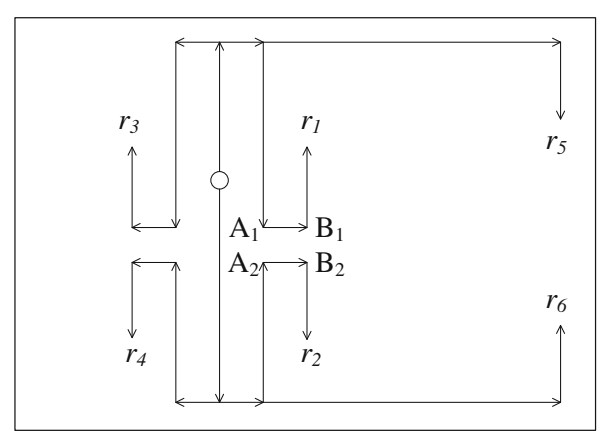

(a) Robots cannot sense boundary

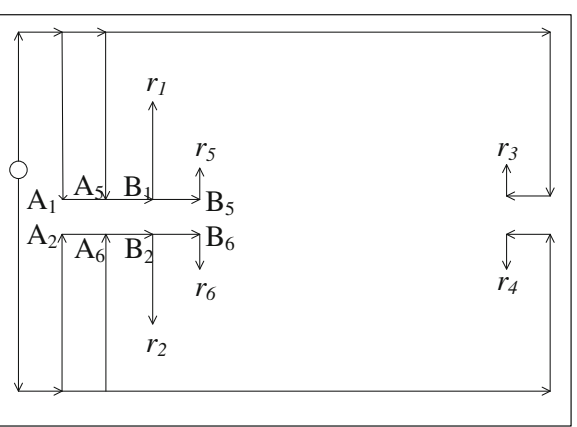

(b) Robots can sense right boundary

Fig. 2 Initialization for exploration discourse of another multi-robot system $(n=6)$; all characters in the figure have the same meanings as in Fig. 1 
Table 1 Notations of predicate logic reasoning

\begin{tabular}{|c|c|c|}
\hline \multirow[t]{10}{*}{ Predicates } & Belonged & A robot is belonged to a subset \\
\hline & Moved & A robot moved along a direction \\
\hline & Travelled & A robot has traveled a distance \\
\hline & Be_oppst & $\begin{array}{l}\text { Moving directions of two robots are } \\
\text { opposite }\end{array}$ \\
\hline & Be_close & Distance between two robots is close \\
\hline & Rcgnz & A robot can recognize the other robot \\
\hline & Fuse & Two robots fuse location information \\
\hline & $\begin{array}{l}\text { Coord }_{x_{1}} \\
\text { coordy }_{y}\end{array}$ & Coordinates $x$ or $y$ of a robot \\
\hline & Avoids & Avoids an object (a robot, or an obstacle) \\
\hline & Turns & Changes current exploring direction \\
\hline \multirow[t]{4}{*}{ Variables } & $r_{i}$ & Robot $r_{i}(i=1, \ldots, n)$ \\
\hline & $S_{k}$ & Subset $k$ of the multi-robot system \\
\hline & $d i r_{i}$ & Moving direction of $r_{i}$ \\
\hline & $d_{r}$ & Rendezvous distance \\
\hline
\end{tabular}

of the two robots should call a collision avoidance subroutine when a rendezvous happened and restores its auxiliary exploring direction after rounding the other robot. The corresponding predicate logic reasoning is

$$
\begin{aligned}
& \text { Belonged }\left(r_{i}, S_{k}\right) \wedge \text { Belonged }\left(r_{j}, S_{m}\right) \wedge \operatorname{Moved}\left(r_{i}, \text { dir }_{i}\right) \wedge \text { Moved } \\
& \left(r_{j}, \text { dir }_{j}\right) \wedge B e_{-} \text {oppst }\left(\text { dir }_{i}, \text { dir }_{j}\right) \wedge B e_{-} \text {close }\left(r_{i}, r_{j}\right) \wedge \\
& \left(\operatorname{Rcgnz}\left(r_{i}, r_{j}\right) \vee \operatorname{Rcgnz}\left(r_{j}, r_{i}\right)\right) \Rightarrow \text { Fuse }\left(\operatorname{Coord}_{x}\left(r_{i}\right)\right. \text {, } \\
& \left.\operatorname{Coord}_{x}\left(r_{j}\right)\right) \wedge \text { Fuse }\left(\operatorname{Coord}_{y}\left(r_{i}\right), \operatorname{Coord}_{y}\left(r_{j}\right)\right) \wedge \text { Avoids }\left(r_{i}, r_{j}\right)
\end{aligned}
$$

If the two robots met on their primary exploring directions, then the two robots turn to their auxiliary directions, respectively, to continue their exploration discourses. If the two auxiliary exploring directions are identical, then location coordinates information fusion is conducted through revised forms of (2) to (6). The revision is obtained by interchanging abscissa and ordinate in (2) to (6). Else, if the two auxiliary exploring directions are different, then no fusion is needed. Upper expression of the rendezvous and corresponding behavior is

$$
\begin{gathered}
\text { Belonged }\left(r_{i}, S_{k}\right) \wedge \text { Belonged }\left(r_{j}, S_{m}\right) \wedge \text { Moved }\left(r_{i}, \text { dir }_{i}\right) \wedge \text { Moved } \\
\left.\left(r_{j}, \text { dir }_{j}\right) \wedge \text { Be_oppst }\left(\operatorname{dir}_{i}, \operatorname{dir}_{j}\right) \wedge \text { Be_close }_{(}, r_{i}\right) \wedge \\
\left(\operatorname{Rcgnz}\left(r_{i}, r_{j}\right) \vee \operatorname{Rcgnz}\left(r_{j}, r_{i}\right)\right) \Rightarrow \text { Fuse }\left(\text { Coord }_{x}\left(r_{i}\right), \text { Coord }_{x}\right. \\
\left.\left(r_{j}\right)\right) \wedge \text { Fuse }\left(\operatorname{Coord}_{y}\left(r_{i}\right), \text { Coord }_{y}\left(r_{j}\right)\right) \wedge \operatorname{Turns}\left(r_{i}, \operatorname{dir}_{i}\right) \wedge \\
\text { Turns }\left(r_{j}, \text { dir }_{j}\right)
\end{gathered}
$$

The behavior for the robot when detecting an obstacle is similar to the case of detecting another robot.
Besides the correction described by (2) to (6) during rendezvous period, a further correction is necessary. The utilized trajectories featured a phenomenon that error accumulated relative to turn location for each robot. Therefore, coordinate $x$ is updated before or after a rendezvous by the following scheme:

$$
\begin{aligned}
& x_{i}^{\prime}(k)=\sum_{j=1}^{k} \mu^{j} x(k-j) / \sum_{j=1}^{k} \mu^{j} \\
& x_{i}(k+1)=x_{i}^{\prime}(k)+\Delta x_{i}(k+1)
\end{aligned}
$$

where $k(k=0,1,2, \ldots)$ is the time instant before or after a rendezvous for $r_{i}$, when a rendezvous begun, ended, or $r_{i}$ turned its exploring direction; $k$ is reset to zeros; and $\mu$ is a scalar, $0<\mu<1$. Correction (10)-(11) need no additional bandwidth of the wireless network.

\section{Experiment settings}

The effectiveness of the proposed approach has been validated by computer simulation experiments. The simulations were conducted in Matlab 7.1 environment on a personal computer which has an Intel $^{\circledR}$ Core $^{\text {TM }}$ i7 $2.93 \mathrm{GHz}$ processor and $8 \mathrm{~GB}$ RAM. It is assumed that the sensing radius is $4 \mathrm{~m}$, and the moving speed is $0.1 \mathrm{~m} / \mathrm{s}$ for all robots.

Dead reckoning error models for robots were taken from [5]. If robot $r_{i}(i=1, \ldots, 6)$ moved along horizontal direction, then the errors along abscissa and ordinate are represented as $E_{a}(k) \leq \xi_{i} D_{i}(k)\left(1-\cos \alpha_{i}\right)$, and $E_{o}(k) \leq$ $\eta_{i} D_{i}(k)\left(1-\sin \alpha_{i}\right)$, respectively, where $D_{i}(k)$ is the total distance traveled by $r_{i}$ along current exploring direction so far, $\alpha_{i}$ is a quite small constant, $k$ is current time instant, and $\xi_{i}=\left[\begin{array}{llllll}1.0 & 1.1 & 1.2 & 1.3 & 1.4 & 1.5\end{array}\right]^{\mathrm{T}}$ and $\eta_{i}=\left[\begin{array}{lll}1.4 & 1.2\end{array}\right.$ $\left.\begin{array}{llll}1.3 & 1.1 & 1.2 & 1.2\end{array}\right]^{\mathrm{T}}$ are coefficient vectors to represent error differences between robots. If $r_{i}$ moved along vertical direction, then the two errors are represented as $E_{a}(k) \leq$ $\eta_{i} D_{i}(k)\left(1-\sin \alpha_{i}\right)$ and $E_{o}(k) \leq \xi_{i} D_{i}(k)\left(1-\cos \alpha_{i}\right)$. All robots initially located at a common position over a $100 \times$ $100 \mathrm{~m}^{2}$ obstacle-free environment, which was modeled as an occupied grid map of $1 \times 1 \mathrm{~m}^{2}$. The initial locations were randomly generated for each run. The robot team size is a variable that ranges from 2 to 6 . For each team size, the simulations were conducted 100 runs.

The proposed error correction strategy was used in multi-robot systems coordinated by predicate logic-based reasoning [12] for unknown environment exploration. Since the size of the individual multi-robot systems is a variable, and exploration times for different system sizes are different too, it is meaningless to compare the total accumulative errors. Therefore, two index functions $e_{o}=\left\|P-\hat{P}_{o}\right\| / n$ and $e_{o c}=\left\|P-\hat{P}_{o c}\right\| / n(n=2, \ldots$, 6) are defined, where $P$ is a vector representing true locations of all robots and $\hat{P}_{o}$ and $\hat{P}_{o c}$ are estimates of 


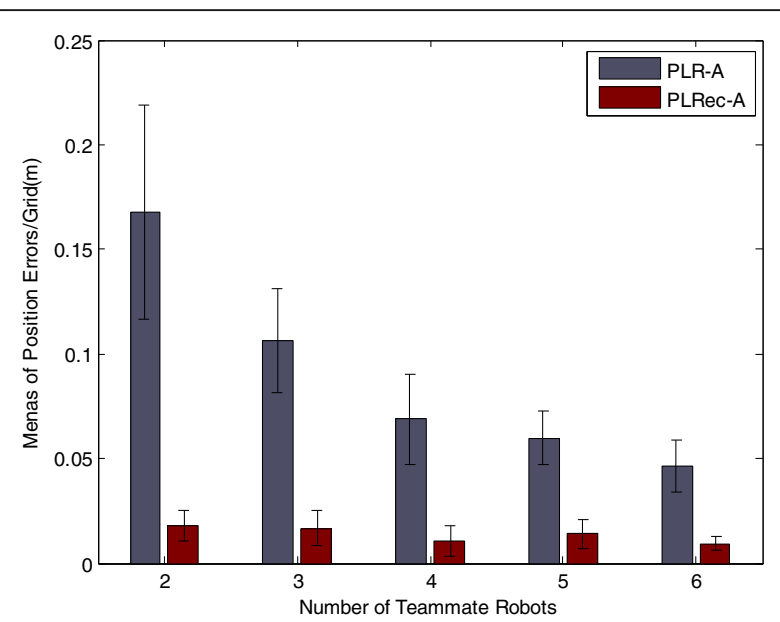

Fig. 3 Localization errors for multi-robot systems of different sizes; in the histogram, left bar and right bar for each team size represent errors of robots coordinated by PLR-A and PLRec-A, respectively

positions for all robots with odometer errors and corrected odometer errors, respectively.

\section{Results and discussions}

\subsection{Simulation and statistical results}

Figure 3 showed the means of $e_{o}$ and $e_{o c}$ per grid on the trajectories during explorations. It can be seen that the proposed PLR-A with error correction (PLRec-A) reduced accumulative errors obviously compared with PLR-A. A special phenomenon for PLRec-A errors in Fig. 3 is that the error for team size 5 is bigger than that for team size 4. This is due to the fact that all robots have their respective cooperative robots for the case of team size being 4 , and $r_{5}$ has no cooperative robot for the case of team size being 5. The corresponding multi-robot systems were partitioned into two and three subsets in two cases, respectively. An overall trend of the errors is that errors descend versus the increasing of multi-robot system size. All robots dispersed over the environment, as discussed in the initialization strategies.

Robot trajectories for a multi-robot system coordinated by PLR-A and PLRec-A, with $n=2$ and initial location is $(41,49)$, are shown in Fig. 4a, b, respectively. It can be seen that dead errors increased along time, and exploration trajectories of robots coordinated by PLRec-A are closer to true trajectories than the trajectories of robots coordinated by PLR-A.

Wilcoxon rank-sum test under significance level $\alpha=$ 0.05 for the two kinds of results are shown in Table 2 . The null hypothesis implies that the error means of populations from which the two samples were taken are equal. If the null hypothesis is rejected, then the error means are different. In the five tests for each system size shown in the table, all standardized values listed in the last row are bigger than 0.776; therefore, the null hypothesis is rejected for all tests, indicating that improvement resulting from PLRec-A are evident.

\subsection{Discussions}

The error correction scheme described in this paper was designed for coordination purpose. A precondition to implement the error correction scheme is that coordinated robots of the multi-robot systems could meet to each other during exploration discourse. In addition, to get a global environment model, postprocessing is necessary $[6,7]$.

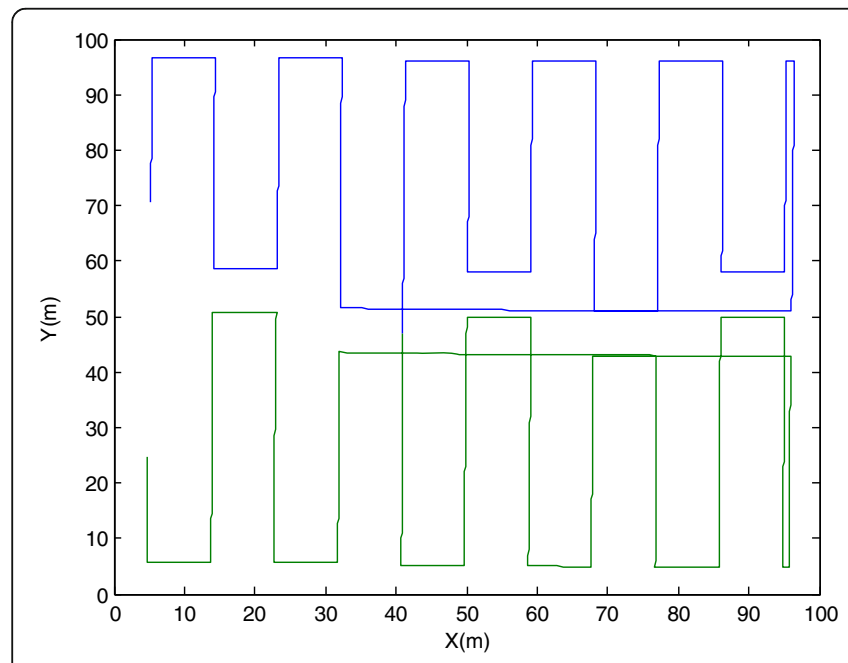

(a) Trajectories with odometry errors

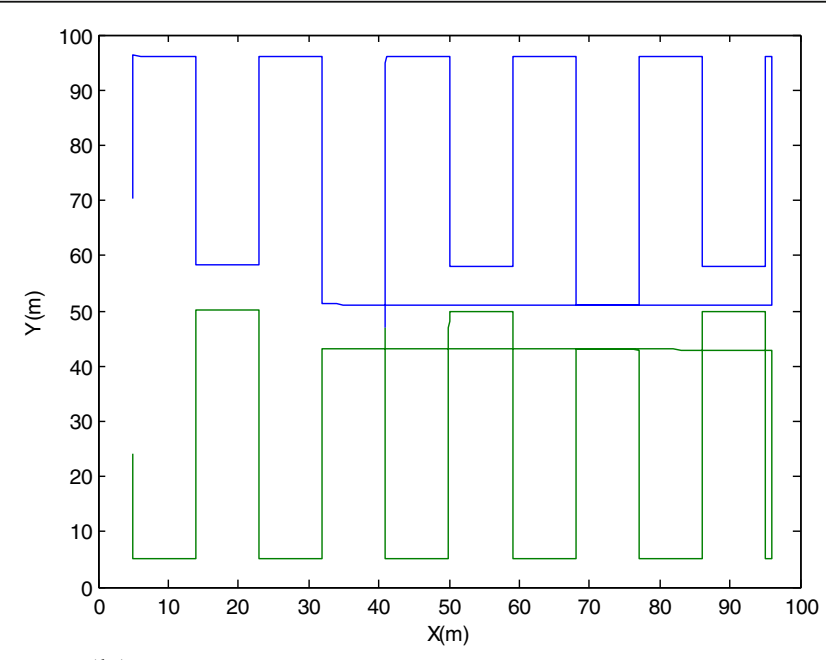

(b) Trajectories with corrected odometry errors

Fig. 4 Exploration trajectories of a multi-robot system composed of two robots; in the figure, blue and green trajectories correspond to $r_{1}$ and $r_{2}$, respectively 
Table 2 Statistical results

\begin{tabular}{llllll}
\hline$n$ & 2 & 3 & 4 & 5 & 6 \\
\hline$\mu_{R_{1}}$ & 10,050 & 10,050 & 10,050 & 10,050 & 10,050 \\
$\sigma_{R_{1}}$ & 167,500 & 167,500 & 167,500 & 167,500 & 167,500 \\
$r_{1}$ & 15,050 & 15,050 & 15,026 & 14,758 & 15,050 \\
$Z$ & 12.217 & 12.217 & 12.158 & 11.504 & 12.217 \\
\hline
\end{tabular}

\section{Conclusions}

A novel uncertainty correction strategy for multi-robot systems in exploring unknown environments has been proposed. The approach adopted a hierarchical architecture to realize error correction. At upper level, the rendezvous was formulated by predicate logic reasoning, and at lower level coordinates, fusion was fused by weighted summation. Although homogeneous multi-robot systems are discussed as default, the results are suitable for heterogeneous multi-robot systems if and only if the widths of zigzag trajectories of the two cooperative robots are kept to be identical. Coordination strategy is a core for multiple robot cooperation. The results proposed in this paper make robust coordination of multi-robot systems under uncertainty becoming possible. Furthermore, additional bandwidth for the wireless network is needed only if rendezvous happened. How to make the proposed approach suitable for dynamic and structured environments is future work.

\section{Abbreviations \\ Dec-iFDD: Decentralized model learning scheme based on the Incremental Feature Dependency Discovery; MacDec-POMDP: Macro-actions decentralized partially observable Markov decision process; MDP: Markov decision process; PLR-A: Predicate logic reasoning-based approach; PLRec-A: PLR-A with error correction; UAV: Unmanned aerial vehicle}

\section{Acknowledgements}

The research presented in this paper was supported by Council of the National Natural Science Foundation of China.

\section{Funding}

The authors acknowledge the National Natural Science Foundation of China (Grant: 61672304).

\section{Authors' contributions}

$X D$ is the main writer of this paper. He proposed the main idea, developed the predicate logic reasoning implications, and conducted the simulation. ZY designed the error correction scheme and analyzed the result. JZ took part in programming for a few of Matlab functions. DL gave some important suggestions for the predicate logic reasoning. All authors read and approved the final manuscript.

\section{Competing interests}

The authors declare that they have no competing interests.

\section{Publisher's Note}

Springer Nature remains neutral with regard to jurisdictional claims in published maps and institutional affiliations.

\section{Author details}

${ }^{1}$ School of Computer and Control Engineering, Qiqihar University, Qiqihar 161006, Heilongjiang, China. ${ }^{2}$ College of Automation, Harbin Engineering University, Harbin 150001, Heilongjiang, China.
Received: 8 March 2018 Accepted: 30 May 2018

Published online: 07 June 2018

\section{References}

1. T Andre, C Bettstetter, Collaboration in multi-robot exploration: to meet or not to meet? J. Intell. Robot. Syst. 82(2), 1-13 (2016)

2. J Banfi, LA Quattrini, I Rekleitis, F Amigoni, N Basilico, Strategies for coordinated multirobot exploration with recurrent connectivity constraints. Auton. Robot. 42(4), 875-894 (2018)

3. T Setter, M M Egerstedt, Energy-constrained coordination of multi-robot teams. IEEE Trans. Control Syst. Technology 25(4), 1257-1263 (2017)

4. A Ivanov, M M Campbell, Joint exploration and tracking: JET. IEEE Control Syst. Lett. 2(1), 43-48 (2018)

5. BS Ryu, HS Yang, Integration of reactive behaviors and enhanced topological map for robust mobile robot navigation. IEEE Trans. Syst. Man Cybern. A Syst. Hum. 29(5), 474-485 (1999)

6. BD Gouveia, D Portugal, C Daniel, DC Silva, L Marques, Computation sharing in distributed robotic systems: a case study on SLAM. IEEE Trans. Autom. Sci. Eng. 12(2), 410-422 (2015)

7. C Estrada, J Neira, TD Tardos, Hierarchical SLAM: real-time accurate mapping of large environments. IEEE Trans. Robot. 21(4), 588-596 (2005)

8. NH Ure, G Chowdhary, YF Chen, JP How, J Vian, Distributed learning for planning under uncertainty problems with heterogeneous teams. J. Intell. Robot. Syst. 74(1-2), 529-544 (2014)

9. C Amato, G Konidaris, A Anders, G Cruz, JP How, LP Kaelbling, Policy search for multi-robot coordination under uncertainty. Int. J. Robot. Res. 35(14), 1760-1778 (2016)

10. J Capitan, MTJ Spaan, L Merino, A Ollero, Decentralized multi-robot cooperation with auctioned POMDPs. Int. J. Robot. Res. 32(6), 650671 (2013)

11. JLS Rincon, P Tokekar, V Kumar, S Carpin, Rapid deployment of mobile robots under temporal, performance, perception, and resource constraints. IEEE Robot. Autom. Lett. 2(4), 2016-2023 (2017)

12. XF Dai, LH Jiang, DH Li, Integrating predicate reasoning and reactive behaviors for coordination of multi-robot systems. Cluster Comput. (2018) https://doi.org/10.1007/s10586-017-1676-8

13. Y Cao, WW Yu, W Wei, GR Chen, An overview of recent progress in the study of distributed multi-agent coordination. IEEE Trans. Indus. Inf 9(1), 427-438 (2013)

14. E Galceran, M Carreras, A survey on coverage path planning for robotics. Robot. Auton. Syst. 61(12), 1258-1276 (2013)

15. L Valentin, R Murrieta-Cid, L Munoz-Gomez, R Lopez-Padilla, M Alencastre-Miranda, Motion strategies for exploration and map building under uncertainty with multiple heterogeneous robots. Adv. Robot. 28(17), 1133-1149 (2014)

16. QR Tang, P Eberhard, Relative observation for multi-robot collaborative localisation based on multi-source signals. J. Exp. Theor. Artif. Intell. 26(4), 571-591 (2014)

17. B Schlotfeldt, D Thakur, N Atanasov, V Kumar, GJ Pappas, Anytime planning for decentralized multi-robot active information gathering. IEEE Robot. Autom. 3(2), 1025-1032 (2018)

18. S Keshmiri, S Payandeh, Regression analysis of multi-rendezvous recharging route in multi-robot environment. Int. J. Soc. Robot. 4(1), 15-27 (2012)

19. N Roy, G Dudek, Collaborative robot exploration and rendezvous: algorithms, performance bounds and observations. Auton. Robot. 11(2), 117-136 (2001)

20. WY Jiang, DB Wang, Y Wang, ZA Ali, UAV rendezvous based on timevarying vector fields. Electronics Lett. 53(10), 653-655 (2017)

21. JJ Cheng, C Liu, MC Zhou, QT Zeng, A Yla-Jaaski, Automatic composition of semantic Web services based on fuzzy predicate Petri nets. IEEE Trans. Autom. Sci. Eng. 12(2), 680-690 (2015)

22. SR Fiorini, J Bermejo-Alonso, P Gonçalves, EP de Freitas, AO Alarcos, JI Olszewska, E Prestes, C Schlenoff, SV Ragavan, S Redfield, B Spencer, H Li, A suite of ontologies for robotics and automation [industrial activities]. IEEE Robot. Autom. Mag. 24(1), 8-11 (2017) 